more axisymmetric. Also, the magnetic field that penetrates the conducting solid inner core can only vary on the much longer magnetic diffusion timescale rather than the shorter timescale of fluid motions in the outer core. This effectively anchors the magnetic field and stabilizes it against the rapid fluctuations in the outer core, allowing the field to maintain a more stable dipole.

If the region interior to the convecting shell is fluid instead of solid, then it can respond to electromagnetic stress through fluid motion and is not constrained by the stabilizing effects of a solid conducting core. As the fluid inner region is not in solid-body rotation, the axisymmetrizing effect of strong differential rotation is suppressed. Also, the magnetic field that penetrates the inner region can vary by moving the fluid instead of only through magnetic diffusion. This reduction in stability, combined with the smaller length scales of the thin shell, promote the creation of higherdegree, non-axisymmetric structure in our numerical models with stably stratified fluid interiors.

As the stabilizing effects of the inner core are a result of its solid state and conductivity, our theory predicts that a dynamo operating in a thin shell surrounding an electrically insulating solid inner core would also produce non-dipolar, non-axisymmetric fields. We have created numerical dynamos operating in a thin shell (similar radius to our convecting shell surrounding the stably stratified interior) surrounding a large solid inner core with a much smaller electrical conductivity than the outer core (inner to outer core conductivity ratios of 0.1-0.0001), and found that they do produce non-dipolar, non-axisymmetric magnetic fields, as predicted.

Future missions to Uranus and Neptune to study further these planets' magnetic fields and internal properties could determine the validity of our model. More accurate surface power spectra extending out to higher degree and order could be produced with magnetic-field measurements acquired closer to the planets with more global spatial distribution. These spectra could then be compared to our numerical model to assess the model's validity. The evolution in time of the magnetic fields could also be compared to our numerical model by examining the changes in the fields since the Voyager II observations. Although no missions to Uranus and Neptune are currently planned, the upcoming data from MESSENGER and Cassini (on the magnetic fields of Mercury and Saturn, respectively) will provide tests of the implication of our results: convective-region geometry is an important factor in determining magnetic-field morphology, albeit in a different context from this study.

Received 9 October 2003; accepted 28 January 2004; doi:10.1038/nature02376.

1. Ness, N. F. et al. Magnetic fields at Uranus. Science 233, 85-89 (1986).

2. Ness, N. F. et al. Magnetic fields at Neptune. Science 246, 1473-1478 (1989).

3. Connerney, J. E. P., Acuna, M. H. \& Ness, N. F. The magnetic field of Uranus. J. Geophys. Res. 92 15329-15336 (1987)

4. Connerney, J. E. P., Acuna, M. H. \& Ness, N. F. The magnetic field of Neptune. J. Geophys. Res. 96, 19023-19042 (1991).

5. Connerney, J. E. P. Magnetic fields of the outer planets. J. Geophys. Res. 98, 18659-18679 (1993).

6. Russell, C. T. Magnetic fields of the terrestrial planets. J. Geophys. Res. 98, 18681-18695 (1993).

7. Ruzmaikin, A. A. \& Starchenko, S. V. On the origin of Uranus and Neptune magnetic fields. Icarus $\mathbf{9 3}$, 82-87 (1991).

8. Podolak, M., Hubbard, W. B. \& Stevenson, D. J. in Uranus (eds Bergstralh, J. T., Miner, E. D. \& Matthews, M. S.) 29-61 (Univ. Arizona Press, Tucson, 1991).

9. Hubbard, W. B., Podolak, M. \& Stevenson, D. J. in Neptune and Triton (ed. Cruikshank, D. P.) 109-138 (Univ. Arizona Press, Tucson, 1995).

10. Holme, R. Three-dimensional kinematic dynamos with equatorial symmetry: application to the magnetic fields of Uranus and Neptune. Phys. Earth Planet. Inter. 102, 105-122 (1997).

11. Hubbard, W. B. et al. Interior structure of Neptune-comparison with Uranus. Science 253, 648-651 (1991).

12. Nellis, W. J. et al. The nature of the interior of Uranus based on studies of planetary ices at high dynamic pressure. Science 240, 779-781 (1988).

13. Kuang, W. \& Bloxham, J. An earth-like numerical dynamo model. Nature 389, 371-374 (1997).

14. Kuang, W. J. \& Bloxham, J. Numerical modelling of magnetohydrodynamic convection in a rapidly rotating spherical shell: weak and strong field dynamo action. J. Comput. Phys. 153, 51-81 (1999).

15. Glatzmaier, G. A. \& Roberts, P. H. A 3-dimensional convective dynamo solution with rotating and finitely conducting inner core and mantle. Phys. Earth Planet. Inter. 91, 63-75 (1995).

16. Glatzmaier, G. A. \& Roberts, P. H. A 3-dimensional self-consistent computer simulation of a geomagnetic field reversal. Nature 377, 203-209 (1995).
17. Dormy, E., Valet, J. P. \& Courtillot, V. Numerical models of the geodynamo and observational constraints. Geochem. Geophys. Geosyst. 1, 2000GC000062 (2000).

18. Grote, E., Busse, F. H. \& Tilgner, A. Regular and chaotic spherical dynamos. Phys. Earth Planet. Inter. $117,259-272(2000)$.

19. Grote, E. \& Busse, F. H. Hemispherical dynamos generated by convection in rotating spherical shells. Phys. Rev. E 62, 4457-4460 (2000).

20. Kutzner, C. \& Christensen, U. R. From stable dipolar towards reversing numerical dynamos. Phys. Earth Planet. Inter. 131, 29-45 (2002).

21. Ishihara, N. \& Kida, S. Equatorial magnetic dipole field intensification by convection vortices in a rotating spherical shell. Fluid Dyn. Res. 31, 253-274 (2002).

22. Aubert, J. \& Wicht, J. Axial versus equatorial dipolar dynamo models with implications for planetary magnetic fields. Earth Planet. Sci. Lett. (in the press).

23. Roberts, P. H. \& Glatzmaier, G. A. The geodynamo, past, present and future. Geophys. Astrophys. Fluid Dynam. 94, 47-84 (2001)

24. Hollerbach, R. \& Jones, C. A. Influence of the Earth's inner core on geomagnetic fluctuations and reversals. Nature 365, 541-543 (1993).

25. Wicht, J. Inner core conductivity in numerical dynamo simulations. Phys. Earth Planet. Inter. 132, 281-302 (2002).

26. Marley, M., Gomez, P. \& Podolak, M. Monte Carlo interior models for Uranus and Neptune. J. Geophys. Res. 100, 23349-23353 (1995).

27. Zhang, K. K. \& Schubert, G. Teleconvection: remotely driven thermal convection in rotating stratified spherical layers. Science 290, 1944-1947 (2000).

28. Jones, C. A., Longbottom, A. W. \& Hollerbach, R. A self-consistent convection driven geodynamo model, using a mean field approximation. Phys. Earth Planet. Inter. 92, 119-141 (1995).

29. Merrill, R. T., McElhinny, M. W. \& McFadden, P. L. The Magnetic Field of the Earth 31 (Academic, San Diego, 1996).

30. Holme, R. \& Bloxham, J. The magnetic fields of Uranus and Neptune: methods and models. J. Geophys. Res. 101, 2177-2200 (1996).

Acknowledgements We thank D. Stevenson for comments on the manuscript. This work was supported by NSERC and NSF

Competing interests statement The authors declare that they have no competing financial interests.

Correspondence and requests for materials should be addressed to S.S.

(stanley@geophysics.harvard.edu).

\section{Observation of entanglement between a single trapped atom and a single photon}

\section{B. B. Blinov, D. L. Moehring, L.- M. Duan \& C. Monroe}

FOCUS Center and University of Michigan Department of Physics, Ann Arbor, Michigan 48109-1120, USA

An outstanding goal in quantum information science is the faithful mapping of quantum information between a stable quantum memory and a reliable quantum communication channel $^{1}$. This would allow, for example, quantum communication over remote distances ${ }^{2}$, quantum teleportation ${ }^{3}$ of matter and distributed quantum computing over a 'quantum internet'. Because quantum states cannot in general be copied, quantum information can only be distributed in these and other applications by entangling the quantum memory with the communication channel. Here we report quantum entanglement between an ideal quantum memory-represented by a single trapped ${ }^{111} \mathrm{Cd}^{+}$ion-and an ideal quantum communication channel, provided by a single photon that is emitted spontaneously from the ion. Appropriate coincidence measurements between the quantum states of the photon polarization and the trapped ion memory are used to verify their entanglement directly. Our direct observation of entanglement between stationary and 'flying' qubits ${ }^{4}$ is accomplished without using cavity quantum electrodynamic techniques ${ }^{5-7}$ or prepared non-classical light sources $^{3}$. We envision that this source of entanglement may be 
used for a variety of quantum communication protocols ${ }^{2,8}$ and for seeding large-scale entangled states of trapped ion qubits for scalable quantum computing 9 .

Atom-photon entanglement has been implicit in many previous experimental systems, from early measurements of Bell inequality violations in atomic cascade systems ${ }^{10,11}$ to fluorescence studies in trapped atomic ions ${ }^{12,13}$. A prime example of current interest is strongly coupled cavity quantum electrodynamics, where individual atoms interact with photons in single-mode cavities ${ }^{5-7}$. Another example is the continuous-variable entanglement between ensembles of atoms and light fields observed in systems containing macroscopic numbers of atoms and photons ${ }^{14-17}$. However, atomphoton entanglement has not been directly observed in previous experiments, as the individual atoms and photons have not been under sufficient control. Here we report the explicit demonstration of qubit entanglement between an individual stationary atom and a 'flying' optical single photon.

In the experiment, a photon is spontaneously emitted from a single trapped atomic ${ }^{111} \mathrm{Cd}^{+}$ion, which is initially excited to a state that has multiple decay channels. Along a certain emission direction selected by an aperture, the photon's polarization is entangled with particular hyperfine ground states in the de-excited atom. The entanglement is directly verified through subsequent polarization analysis of the photon and state detection of the trapped ion. Given the small acceptance angle of the aperture and other losses, the probability $P$ of detecting a photon in a given trial is small. This is

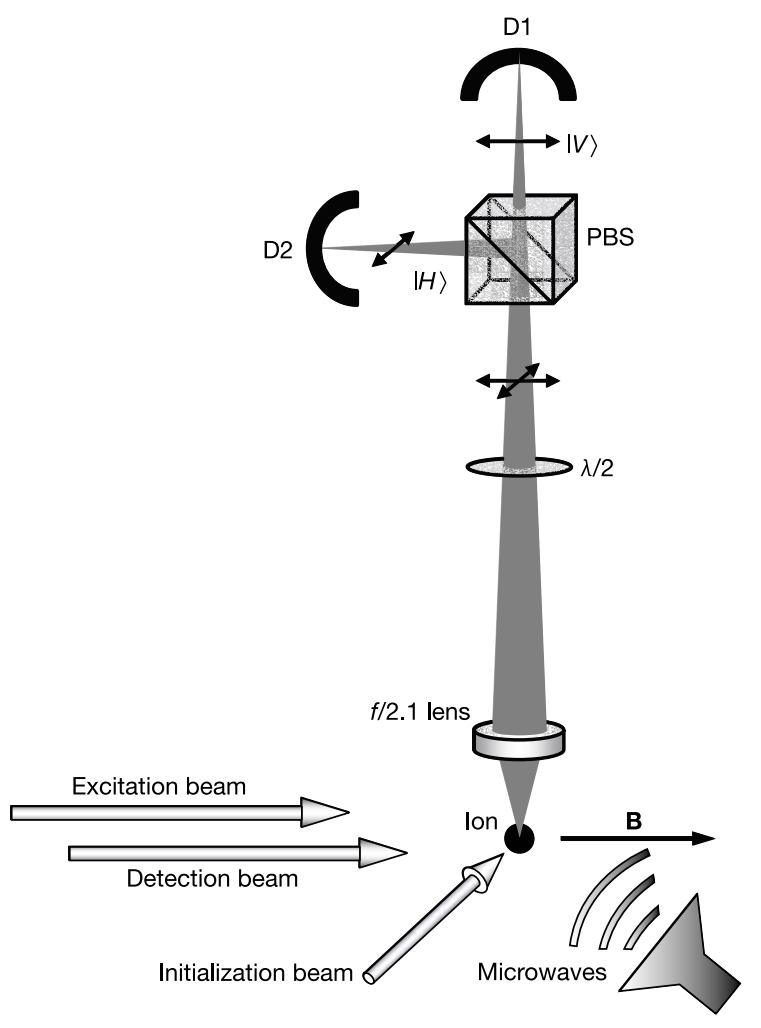

Figure 1 The experimental apparatus. The $\boldsymbol{\pi}$-polarized initialization beam propagates perpendicular to the quantization axis defined by a magnetic field $\mathbf{B}$, while the $\sigma^{+}$-polarized excitation and detection beams propagate along the quantization axis. The scattered photons are collected by an imaging lens and directed to a polarizing beamsplitter (PBS), and two photon-counting detectors D1 and D2 register the $\mathrm{V}$ - and $\mathrm{H}$-polarized photons, respectively. The $\mathrm{\lambda} / 2$-waveplate is used to rotate the photon polarization for photonic qubit measurements in different bases. A microwave horn is located near the trap to drive coherent transitions between the hyperfine levels of the atomic ground state, at a frequency near $14.5 \mathrm{GHz}$. reminiscent of the production of entangled photon pairs through spontaneous optical parametric down-conversion ${ }^{3}$, but in the current system one of the two daughter qubits resides within a trapped atomic ion-perhaps the most reliable of all qubit memories $^{18}$. When accompanied by conventional quantum gates between local trapped ions ${ }^{19-21}$, this probabilistic entanglement protocol can form the basis for a scalable architecture for quantum communication $^{2}$ and computation'.

A diagram of the experimental apparatus is shown in Fig. 1. We trap and laser cool a single ${ }^{111} \mathrm{Cd}^{+}$ion in an asymmetric-quadrupole radio frequency trap with a characteristic size of about $0.7 \mathrm{~mm}$. (In this experiment, it is not necessary to laser cool the ion to the Lamb-Dicke limit.) Several laser pulses tuned near the ${ }^{2} \mathrm{~S}_{1 / 2}{ }^{2} \mathrm{P}_{3 / 2}$ atomic resonance at $214.5 \mathrm{~nm}$ (1) initialize the internal atomic qubit state, (2) excite the atom for the subsequent spontaneous emission of a photon, and (3) detect the internal state of the atom. An applied magnetic field of $B \approx 0.7 \mathrm{G}$ provides a quantization axis for definition of the photon polarization and the internal atomic qubit levels, stored in ${ }^{2} S_{1 / 2}$ hyperfine ground states denoted by quantum numbers $\left|F, m_{F}\right\rangle$, where $F$ is the total angular momentum and $m_{F}$ is its projection along the quantization axis.

Figure 2 shows a diagram of the relevant energy levels of a ${ }^{111} \mathrm{Cd}^{+}$ atomic ion, along with the step-by-step description of the experimental procedure. A short $\pi$-polarized laser pulse followed by a resonant microwave transfer pulse initializes the ion in the $|1,0\rangle$ state (Fig. 2a). A 50-ns pulse of $\sigma^{+}$-polarized laser light weakly excites the ion to the ${ }^{2} \mathrm{P}_{3 / 2}|2,1\rangle$ state, which has a radiative lifetime of $\tau_{\mathrm{e}} \approx 3 \mathrm{~ns}$. The ion then spontaneously decays to either the ${ }^{2} \mathrm{~S}_{1 / 2}$ $|1,1\rangle$ ground state (defined as $|\downarrow\rangle$ ) while emitting a $\pi$-polarized photon, or the ${ }^{2} S_{1 / 2}|1,0\rangle$ ground state (defined as $|\uparrow\rangle$ ) while emitting a $\sigma^{+}$-polarized photon (Fig. 2b). The single photon pulses are collected with an $f / 2.1$ imaging lens whose axis is perpendicular to the quantization axis. Along this direction, the states of polarization of the $\sigma^{+}$and the $\pi$ photons are orthogonal: the former (defined as $|H\rangle$ ) is polarized perpendicular to the quantization axis,

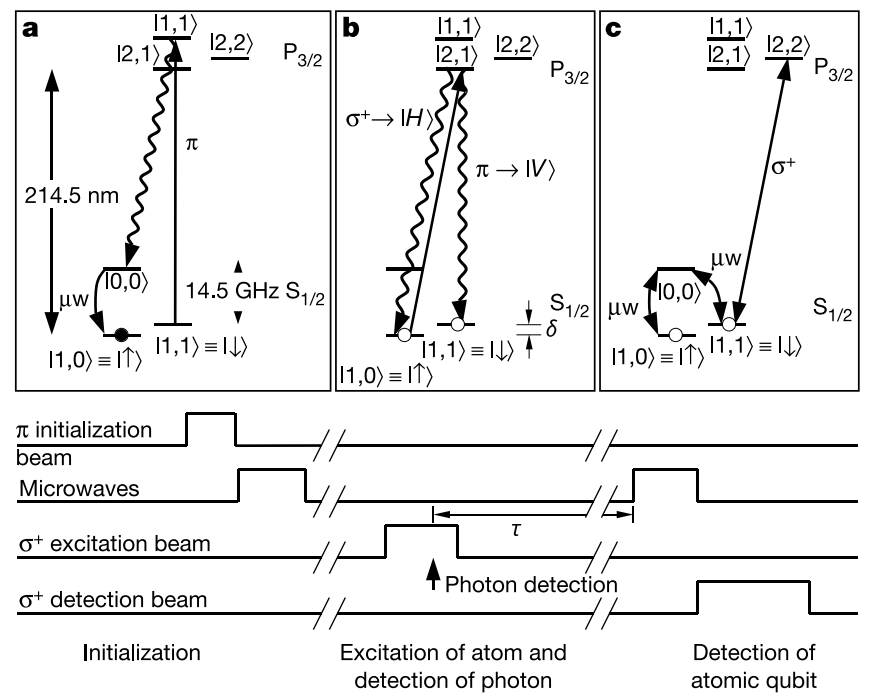

Figure 2 The experimental procedure (time axis not to scale). $\mathbf{a}$, The atomic qubit is initialized to the $|\uparrow\rangle$ hyperfine ground state, following a $30-\mu \mathrm{s} \pi$-polarized optical pumping pulse and a $15-\mu \mathrm{s}$ microwave $(\mu \mathrm{w})$ rotation. $\mathbf{b}$. The atom is weakly excited with a 50-ns $\sigma^{+}$-polarized optical pulse, resulting in spontaneous emission to either state $|\uparrow\rangle$ or state $|\downarrow\rangle$ (separated by frequency $\delta \approx 2 \pi(1.0 \mathrm{MHz})$ ), accompanied by emission and detection of a photon polarized in state $|H\rangle$ or state $|V\rangle$, respectively. c. After a delay of $\tau \approx 1 \mu \mathrm{s}$, a $15-\mu \mathrm{s}$ microwave rotation pulse prepares the atomic qubit for measurement in any basis, and finally a $200-\mu \mathrm{S} \sigma^{+}$-polarized optical detection pulse provides efficient measurement of the atomic qubit. 
while the latter (defined as $|V\rangle$ ) is polarized along the quantization axis, as described in the Methods section. The collected light passes through a polarization rotator (a $\lambda / 2$ waveplate) followed by a polarizing beamsplitter. The two polarization components are then directed to photon-counting photomultiplier tubes (PMTs), each of quantum efficiency $\eta \approx 20 \%$. Following a single photon detection on either PMT, a microwave rotation is applied to the atom, which prepares the atomic qubit to be measured in any basis. This measurement is performed with a $\sigma^{+}$-polarized detection pulse following standard trapped ion fluorescence techniques ${ }^{22}$ (Fig. 2c), with an atomic qubit detection efficiency greater than $95 \%$.

The atomic and photonic qubits are entangled following the spontaneous emission because there are two decay channels from the ${ }^{2} \mathrm{P}_{3 / 2}|2,1\rangle$ excited state, each resulting in distinct photon polarizations. In the experiment, these decay channels are equally likely, but the entanglement is not perfect, as the intensity of the radiation patterns for the $\pi$-polarized photons and for the $\sigma^{+}$polarized photons differ by a factor of two along the viewing axis. Thus, the resulting state created upon a photon emission in this direction is $\sqrt{\frac{1}{3}}|H \uparrow\rangle+\sqrt{\frac{2}{3}}|V \downarrow\rangle$, which exhibits an entanglement fidelity of 0.97 under ideal conditions. This entanglement fidelity can (in principle) be made perfect by simply inserting a polarization-selective lossy element into the path of the photons, at the cost of a somewhat lower efficiency ${ }^{23}$. Unit fidelity can also be achieved by detecting the scattered photons along the quantization axis, with the initial atom excitation to a $\left|m_{F}=0\right\rangle$ excited state, such that only $\sigma^{+}$- and $\sigma^{-}$-polarized photons are collected. We avoid this option, however, as it would require placement of PMTs in a direct line-ofsight with the atomic qubit detection laser beam.

We first measure the conditional probabilities of detecting a certain atomic qubit state given the photonic qubit state after $\sim 1,000$ successful trials. These probabilities are plotted in Fig. 3, with $P(\uparrow \mid H)=0.97 \pm 0.01, P(\downarrow \mid H)=0.03 \pm 0.01, P(\uparrow \mid V)=0.06 \pm$ 0.01 and $P(\downarrow \mid V)=0.94 \pm 0.01$, where the errors are statistical. To verify entanglement, we repeat the correlation measurement in a different basis of both photonic and atomic qubits. The photon polarization is rotated by $45^{\circ}$ using the $\lambda / 2$ waveplate, and the atomic qubit is rotated by applying microwaves driving the $|10\rangle-|00\rangle$ and the $|11\rangle-|00\rangle$ transitions as indicated in Fig. 2c. Both qubit rotations are through a Bloch polar angle of $\pi / 2$, and the relative phase of the photonic versus atomic qubit rotations is given by $\phi=\delta \tau+\phi_{\mu}$, where $\delta \approx 2 \pi(1.0 \mathrm{MHz})$ is the Zeeman splitting between the $|1,0\rangle$ and the $|1,1\rangle$ levels, and $\tau \approx 1 \mu \mathrm{s}$ is the

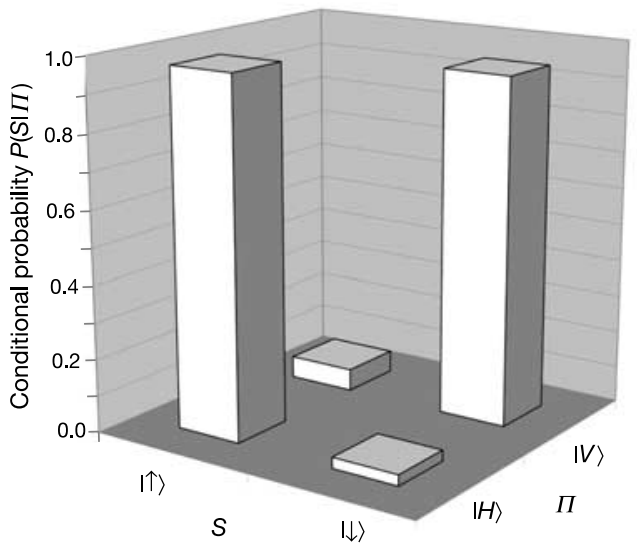

Figure 3 Measured conditional probabilities in the original basis (no atomic or photonic qubit rotation before measurement). The bars indicate the probabilities $P(S \mid \Pi)$ of detecting atomic qubit states $S=\uparrow$ or $\downarrow$ conditioned upon detecting photon qubit states $\Pi=H$ or $V$. time delay between the photon emission and the application of the microwave pulse of phase $\phi_{\mu}$. Varying the relative phase of the two qubit rotations by adjusting $\phi_{\mu}$ produces the correlation fringes shown in Fig. 4a. Figure $4 \mathrm{~b}$ shows the values of conditional probabilities at the point of maximum correlation; these probabilities are $P(\uparrow \mid H)=0.89 \pm 0.01, P(\downarrow \mid H)=0.11 \pm 0.01, P(\uparrow \mid V)=$ $0.06 \pm 0.01$ and $P(\downarrow \mid V)=0.94 \pm 0.01$. If the atomic and photonic qubits were not entangled but instead prepared in a statistical mixture, then all of these conditional probabilities would have been 0.5 .

From these measured correlations, we calculate a bound on the entanglement fidelity to be $F \geq 0.87$ (described in the Methods section), somewhat lower than the potential 0.97 fidelity described above, but still significantly larger than the entanglement threshold of $F>0.5$ (ref. 24). Several factors contribute to this decrease in fidelity, including: multiple excitations of the atom during the pump pulse $(2.5 \%)$, mixing of the photon polarizations owing to the nonzero solid angle $(0.5 \%)$, imperfect rotations of the atomic qubit, mainly due to a 50-ns jitter in the delay $\tau(1.5 \%)$, background counts and dark counts on the PMTs leading to false positives (5-10\%), and imperfections in the polarizing beamsplitter $(3 \%)$. We estimate that magnetic field fluctuations affecting the atomic qubit reduce the fidelity by $\ll 1 \%$. All sources of errors combine to give a $\sim 9 \%$ reduction of entanglement fidelity, consistent with the observation.

The success probability $P$ of creating an entangled state in a given trial depends on the efficiency of generating a single photon and
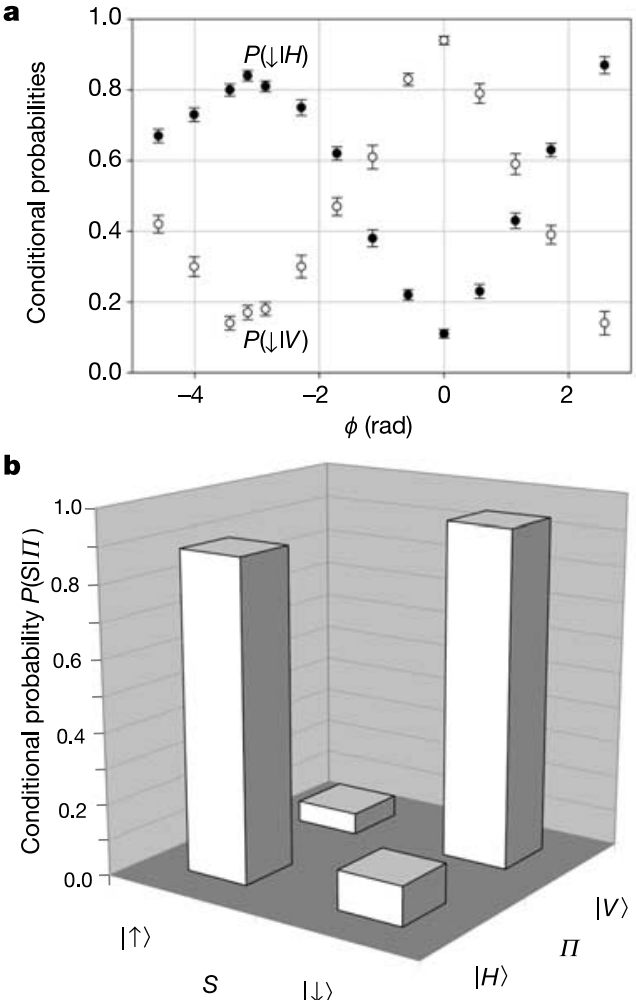

Figure 4 Conditional probabilities after both atomic and photonic qubits are rotated by a polar angle of $\pi / 2$ in the Bloch sphere. a, Measured conditional probabilities $P(\downarrow \mid H)$ and $P(\downarrow \mid V)$ as the relative phase $\phi$ between the atomic and photonic rotations is varied. b. Measured conditional probabilities $P(S \mid \Pi)$ at the point of highest correlation, defined as $\phi=0$. If the atomic and photonic qubits were not entangled but instead prepared in a statistically mixed state, then all conditional probabilities in the figure would have been 0.5 . 
then detecting the emitted photon. In our excitation scheme the probability of emitting a single photon in each trial is $P_{\text {exc }} \approx 0.1$ to suppress the multiple-excitation rate. The efficiency of a single photon detection in turn depends on the light collection solid angle $\Delta \Omega$, the transmission efficiency $T$ of the optical elements, and the quantum efficiency of the detectors themselves. The success probability is $P=\eta T P_{\text {exc }}(\Delta \Omega / 4 \pi) \approx(0.2)(0.4)(0.1)(0.02) \approx 1.6 \times 10^{-4}$. The experiment repetition rate is $R \approx 2 \times 10^{3} \mathrm{~s}^{-1}$, resulting in an entanglement generation rate $R_{1}=P R \approx 0.3 \mathrm{~s}^{-1}$.

Several improvements could significantly increase this yield. The repetition rate $R$ could approach the excited-state spontaneous emission rate of $1 / \tau_{\mathrm{e}} \approx 10^{8} \mathrm{~s}^{-1}$. Using a fast, tailored laser pulse for the excitation could push $P_{\text {exc }}$ towards unity, while eliminating multiple excitations. In addition, an imaging lens with a larger numerical aperture would improve the collection solid angle. A trade-off here is that higher collection efficiency comes at the cost of lower fidelity of the entangled state, which can be shown to vary as $F=0.97-0.24(\Delta \Omega / 4 \pi)$ for $\Delta \Omega \ll 4 \pi$. An alternative is to surround the ion with an optical cavity ${ }^{25,26}$ that would allow the collection of most of the photons scattered in each experiment and effectively make $\Delta \Omega / 4 \pi$ approach unity without sacrificing fidelity. It is important to note that this cavity need not be in the strong-coupling regime, as it affects only the entanglement efficiency, not the fidelity.

The atom-photon entanglement observed here may allow the generation of entangled states of remotely located trapped ion qubits, as follows. Consider two distant trapped ion qubits, each of which becomes entangled with their respective photons in the above fashion. When the emitted photons are mode-selected and combined on a beam splitter, appropriate coincidence measurements of the photons ensure the entanglement of the two trapped ion memories ${ }^{27-29}$. The success probability for this twin event may be small (of order $P^{2}$ ), but when the entanglement eventually occurs after many trials, success is known, and this entanglement can be used for further scalable quantum information processing ${ }^{9}$. Given the low success probability $P$ per trial in the current set-up, the rate of generating the two-ion entanglement would be $R_{2}=P^{2} R \approx$ $10^{-4} \mathrm{~s}^{-1}$. However, with the improvements of fast excitation discussed above, and employing better optics, this rate could approach $R_{2} \approx 10^{3} \mathrm{~s}^{-1}$ (assuming a $100-\mathrm{MHz}$ experiment repetition rate). Finally, if reasonable-quality cavities were to be installed around each atomic qubit, this rate could approach $R_{2} \approx 10^{6} \mathrm{~s}^{-1}$.

\section{Methods}

\section{Polarization state of spontaneous emission along arbitrary axis}

The polarization state of photons spontaneously emitted from an atomic dipole depends upon the change in angular momentum $\Delta m$ of the atom along the dipole axis, and the direction of the emission, following the appropriate dipole radiation pattern. For $\Delta m=0$, the (unnormalized) polarization state of a spontaneously emitted photon is $\left|\Pi_{0}\right\rangle=$ $-\sin \theta|\hat{\theta}\rangle$ and for $\Delta m= \pm 1$, the states are $\left|\Pi_{ \pm 1}\right\rangle=\frac{\mathrm{e}^{ \pm i \varphi}}{\sqrt{2}}(\cos \theta|\hat{\theta}\rangle \pm i|\hat{\varphi}\rangle)$, where $\theta$ and $\varphi$ are the spherical polar and azimuthal angles of the emitted photon with respect to the dipole axis, and $\hat{\theta}$ and $\hat{\varphi}$ are their associated spherical coordinate unit vectors. Note that when a photon is emitted perpendicular to the dipole axis $(\theta=\pi / 2)$, the $\Delta m= \pm 1$ radiation is linearly polarized and orthogonal to the $\Delta m=0$ radiation. For multiple decay channels of spontaneous emission to different states of atomic angular momentum $\left|S_{\Delta m_{i}}\right\rangle$, the resulting (unnormalized) state of photon and atom is $\Psi=\sum C_{\Delta m_{i}}\left|\Pi_{\Delta m_{i}}\right\rangle\left|S_{\Delta m_{i}}\right\rangle$, where $C_{\Delta m_{i}}$ are atomic Clebsch-Gordon (CG) coefficients.

In the current experiment, spontaneous emission occurs via a $\Delta m=-1$ transition to atomic state $\left|S_{-1}\right\rangle \equiv|\downarrow\rangle$ and via a $\Delta m=0$ transition to atomic state $\left|S_{0}\right\rangle \equiv|\uparrow\rangle$, with identical CG coefficients, resulting in the final (normalized) state of the emitted photon and atom:

$$
\Psi=\frac{-\sqrt{2} \sin \theta|\hat{\theta}\rangle|\uparrow\rangle+e^{-i \varphi} \cos \theta|\hat{\theta}\rangle|\downarrow\rangle-i \mathrm{e}^{-i \varphi}|\hat{\varphi}\rangle|\downarrow\rangle}{\sqrt{2+\sin ^{2} \theta}}
$$

Along a viewing axis orthogonal to the dipole $(\theta=\pi / 2)$, this state is highly entangled. In addition to this example, there are many other configurations that result in atomphoton entanglement.

\section{Determining entanglement fidelity}

The entanglement fidelity of an arbitrary two-qubit quantum state can be written as its overlap with an appropriate maximally entangled two-qubit state ${ }^{30}$. In the current experiment, the two qubits are represented by a $4 \times 4$ density matrix $\rho$ with photon/ atomic basis states $|H \uparrow\rangle,|H \downarrow\rangle,|V \uparrow\rangle$ and $|V \downarrow\rangle$. The entanglement fidelity with respect to the particular maximally entangled state $\left|\Psi_{M E}\right\rangle=\frac{1}{\sqrt{2}}(|H \uparrow\rangle+|V \downarrow\rangle)$ is given by:

$$
F=\left\langle\Psi_{M E}|\rho| \Psi_{M E}\right\rangle=\frac{1}{2}\left(\rho_{H \dagger, H \uparrow}+\rho_{V \downarrow, V \downarrow}+\rho_{H \uparrow, V \downarrow}+\rho_{V \downarrow, H \dagger}\right)
$$

where $\rho_{\Pi S, \Pi^{\prime} S^{\prime}}=\left\langle\Pi S|\rho| \Pi^{\prime} S^{\prime}\right\rangle$ with $\Pi=H$ or $V$ and $S=\uparrow$ or $\downarrow$. (This expression also holds for any maximally entangled target state by appropriately redefining the basis states.) The first two terms in this expression are the measured correlation probabilities of detecting state $|H\rangle$ with $|\uparrow\rangle$ and state $|V\rangle$ with $|\downarrow\rangle$. The last two coherence terms can be determined by repeating the experiment while independently rotating each qubit through a polar angle of $\pi / 2$ in the Bloch sphere before measurement. The rotated quantum state is then given by $\tilde{\rho}=R_{\pi / 2}(\phi) \rho R_{\pi / 2}^{\dagger}(\phi)$, where $R_{\pi / 2}(\phi)$ is a $\pi / 2$ polar rotation operator for both qubits with relative phase $\phi$. We find that:

$$
\begin{aligned}
\mathrm{e}^{-i \phi} \rho_{H \uparrow, V \downarrow}+\mathrm{e}^{i \phi} \rho_{V \downarrow, H \uparrow} & =\tilde{\rho}_{H \uparrow, H \uparrow}+\tilde{\rho}_{V \downarrow, V \downarrow}-\tilde{\rho}_{H \downarrow, H \downarrow}-\tilde{\rho}_{V \uparrow, V \uparrow}-\left(e^{-i \phi} \rho_{V \uparrow, H \downarrow}+\mathrm{e}^{i \phi} \rho_{H \downarrow, V \uparrow}\right) \\
& \geq \tilde{\rho}_{H \uparrow, H \uparrow}+\tilde{\rho}_{V \downarrow, V \downarrow}-\tilde{\rho}_{H \downarrow, H \downarrow}-\tilde{\rho}_{V \uparrow, V \uparrow}-2 \sqrt{\rho_{H \downarrow, H \downarrow} \rho_{V \uparrow, V \uparrow}}
\end{aligned}
$$

so that a lower bound on the entanglement fidelity can be expressed in terms of diagonal density matrix elements in the original and rotated basis (with $\phi$ set to zero):

$$
F \geq \frac{1}{2}\left(\rho_{H \uparrow, H \uparrow}+\rho_{V \downarrow, V \downarrow}-2 \sqrt{\rho_{H \downarrow, H \downarrow} \rho_{V \uparrow, V \uparrow}}+\tilde{\rho}_{H \uparrow, H \uparrow}+\tilde{\rho}_{V \downarrow, V \downarrow}-\tilde{\rho}_{H \downarrow, H \downarrow}-\tilde{\rho}_{V \uparrow, V \uparrow}\right)
$$

These diagonals are expressed in terms of the measured probabilities as:

$$
\rho_{\Pi S, \Pi S}=P(S \mid \Pi) P(\Pi) \text { and } \tilde{\rho}_{\Pi S, \Pi S}=\tilde{P}(S \mid \Pi) \tilde{P}(\Pi)
$$

For fidelities $F>0.5$, the underlying quantum state is entangled ${ }^{24}$.

Received 19 December 2003; accepted 30 January 2004; doi:10.1038/nature02377.

1. DiVincenzo, D. The physical implementation of quantum computation. Fortschr. Phys. 48, 771-783 (2000).

2. Duan, L.-M., Lukin, M., Cirac, J. I. \& Zoller, P. Long-distance quantum communication with atomic ensembles and linear optics. Nature 414, 413-418 (2001).

3. Bouwmeester, D., Ekert, A. \& Zeilinger, A. (eds) Quantum Cryptography, Quantum Teleportation, Quantum Computation (Springer, Springer, 2000).

4. Gheri, K., Ellinger, K., Pellizzari, T. \& Zoller, P. Photon-wavepackets as flying quantum bits. Fortschr. Phys. 46, 401-415 (1998).

5. Haroche, S., Raimond, J. M. \& Brune, M. in Experimental Quantum Computation and Information (eds de Martini, F. \& Brune, M.) 3-36 (Proc. Int. School of Physics Enrico Fermi, course CXLVIII, IOS Press, Amsterdam, 2002)

6. Kuhn, A. \& Rempe, G. in Experimental Quantum Computation and Information (eds de Martini, F. \& Monroe, C.) 37-66 (Proc. Int. School of Physics Enrico Fermi, course CXLVIII, IOS Press, Amsterdam, 2002).

7. McKeever, J. et al. State-insensitive cooling and trapping of single atoms in an optical cavity. Phys. Rev. Lett. 90, 133602 (2003).

8. Briegel, H.-J., Duer, W., Cirac, J. I. \& Zoller, P. Quantum repeaters: the role of imperfect local operations in quantum communication. Phys. Rev. Lett. 81, 5932-5935 (1998).

9. Duan, L.-M., Blinov, B. B., Moehring, D. L. \& Monroe, C. Scalable trapped ion quantum computation with a probabilistic ion-photon mapping. Preprint at $\langle$ http://www.arxiv.org/quant-ph/0401020 (2004).

10. Freedman, S. J. \& Clauser, J. F. Experimental test of local hidden variables theories. Phys. Rev. Lett. 28, 938-941 (1972).

11. Aspect, A., Grangier, P. \& Roger, G. Experimental realization of Einstein-Podolsky-Rosen-Bohm Gedanken experiment: a new violation of Bell's inequalities. Phys. Rev. Lett. 49, 91-94 (1982).

12. Eichmann, U. et al. Young's interference experiment with light scattered from two atoms. Phys. Rev. Lett. 70, 2359-2362 (1993).

13. DeVoe, R. G. \& Brewer, R. G. Observation of superradiant and subradiant spontaneous emission of two trapped ions. Phys. Rev. Lett. 76, 2049-2052 (1996).

14. Kuzmich, A., Mandel, L. \& Bigelow, N. Generation of spin squeezing via continuous quantum nondemolition measurement. Phys. Rev. Lett. 85, 1594-1597 (2000).

15. Julsgaard, B., Kozhekin, A. \& Polzik, E. Experimental long-lived entanglement of two macroscopic objects. Nature 413, 400-403 (2001).

16. Kuzmich, A. et al. Generation of nonclassical photon pairs for scalable quantum communication with atomic ensembles. Nature 423, 731-734 (2003).

17. van der Wal, C. H. et al. Atomic memory for correlated photon states. Science 301, 196-200 (2003).

18. Wineland, D. J. et al. Experimental issues in coherent quantum manipulation of trapped atomic ions. NIST J. Res 103, 259-328 (1998).

19. Cirac, J. I. \& Zoller, P. Quantum computations with cold trapped ions. Phys. Rev. Lett. 74, 4091-4094 (1995).

20. Sørensen, A. \& Mølmer, K. Quantum computation with ions in thermal motion. Phys. Rev. Lett. 82, 1971-1975 (1999).

21. García-Ripoll, J. J., Zoller, P. \& Cirac, J. I. Speed optimized two-qubit gates with laser coherent control techniques for ion trap quantum computing. Phys. Rev. Lett. 91, 157901 (2003).

22. Blatt, R. \& Zoller, P. Quantum jumps in atomic systems. Eur. J. Phys. 9, 250-256 (1988).

23. Badzięg, P., Horodecki, M., Horodecki, P. \& Horodecki, R. Local environment can enhance fidelity of quantum teleportation. Phys. Rev. A 62, 012311 (2000).

24. Sackett, C. A. et al. Experimental entanglement of four particles. Nature 404, 256-259 (2000).

25. Guthorlein, G., Keller, M., Hayasaka, H., Lange, W. \& Walther, H. A single ion as a nanoscopic probe of an optical field. Nature 414, 49-51 (2001).

26. Mundt, A. B. et al. Coupling a single atomic quantum bit to a high finesse optical cavity. Phys. Rev. Lett. 89, 103001 (2002).

27. Duan, L.-M. \& Kimble, J. Efficient engineering of multiatom entanglement through single-photon detections. Phys. Rev. Lett. 90, 253601 (2003). 
28. Simon, C. \& Irvine, W. Robust long-distance entanglement and a loophole-free Bell test with ions and photons. Phys. Rev. Lett. 91, 110405 (2003).

29. Cabrillo, C., Cirac, J. I., Garcia-Fernandez, P. \& Zoller, P. Creation of entangled states of distant atoms by interference. Phys. Rev. A 59, 1025-1033 (1999).

30. Bennett, C. H., DiVincenzo, D. P., Smolin, J. A. \& Wootters, W. K. Mixed-state entanglement and quantum error correction. Phys. Rev. A 54, 3824-3851 (1996).

Acknowledgements We acknowledge discussions with M. Madsen, P. Haljan, M. Acton and D. Wineland, and thank R. Miller for assistance in building the trap apparatus. This work was supported by the National Security Agency, the Advanced Research and Development Activity, under Army Research Office contract, and the National Science Foundation Information Technology Research Division.

Competing interests statement The authors declare that they have no competing financial interests.

Correspondence and requests for materials should be addressed to B.B. (bblinov@umich.edu).

\section{Supramolecular dendritic liquid quasicrystals}

\section{Xiangbing Zeng ${ }^{1}$, Goran Ungar ${ }^{1}$, Yongsong Liu ${ }^{1}$, Virgil Percec ${ }^{2}$, Andrés E. Dulcey ${ }^{2}$ \& Jamie K. Hobbs}

${ }^{1}$ Department of Engineering Materials, University of Sheffield, Sheffield S1 3JD, UK

${ }^{2}$ Roy \& Diana Vagelos Laboratories, Department of Chemistry, University of Pennsylvania, Philadelphia, Pennsylvania 19104-6323, USA

${ }^{3}$ H. H. Wills Physics Laboratory, University of Bristol, Bristol BS8 1TL, UK

A large number of synthetic and natural compounds selforganize into bulk phases exhibiting periodicities on the $10^{-8}$ $10^{-6}$ metre scale ${ }^{1}$ as a consequence of their molecular shape, degree of amphiphilic character and, often, the presence of additional non-covalent interactions. Such phases are found in lyotropic systems ${ }^{2}$ (for example, lipid-water, soap-water), in a range of block copolymers ${ }^{3}$ and in thermotropic (solvent-free) liquid crystals ${ }^{4}$. The resulting periodicity can be one-dimensional (lamellar phases), two-dimensional (columnar phases) or three dimensional ('micellar' or 'bicontinuous' phases). All such twoand three-dimensional structures identified to date obey the rules of crystallography and their symmetry can be described, respectively, by one of the 17 plane groups or 230 space groups. The 'micellar' phases have crystallographic counterparts in transition-metal alloys, where just one metal atom is equivalent to a $10^{3}-10^{4}$-atom micelle. However, some metal alloys are known to defy the rules of crystallography and form so-called quasicrystals, which have rotational symmetry other than the allowed two-, three-, four- or six-fold symmetry ${ }^{5}$. Here we show that such quasiperiodic structures can also exist in the scaled-up micellar phases, representing a new mode of organization in soft matter.

Research on bulk nanoscale self-assembly of organic matter is partly motivated by the fact that such complex structures may serve as scaffolds for photonic materials ${ }^{6}$ and other nanoarrays, or as precursors for mesoporous ceramics or elements for molecular electronics. Larger biological objects, such as cylinder-like or sphere-like viruses, also pack on similar macrolattices ${ }^{7}$.

Dendrons and dendrimers (tree-like molecules ${ }^{8}$ ) are proving particularly versatile in generating periodic nanostructures (Fig. 1). Two micellar lattices, with space groups $\operatorname{Im} \overline{3} m$ (bodycentred cubic, b.c.c.) ${ }^{9}$, and $P m \overline{3} n$ (refs 10, 11), have been established. An analogue of the $\operatorname{Im} \overline{3} m$ phase has also been observed in block copolymers ${ }^{12}$, and that of the $P m \overline{3} n$ phase in lyotropic liquid crystals $^{13}$. Recently, a complex three-dimensional (3D) tetragonal lattice (space group $P 4_{2} / \mathrm{mnm}$ ) was found, having 30 self-assembled micelles in the unit cell (Fig. 1f) ${ }^{14}$.

In many dendron systems, thermal transitions between the phases in Fig. 1 occur. The master sequence $\mathrm{Col}_{h} \rightarrow P m \overline{3} n \rightarrow$ $\mathrm{P}_{2} / \mathrm{mnm} \rightarrow \operatorname{Im} \overline{3} \mathrm{~m}$ is obeyed with increasing temperature; in only a handful of cases are all these phases displayed in the same material. In a number of compounds, however, an additional unidentified phase has been observed below any other $3 \mathrm{D}$ phase but above $\mathrm{Col}_{\mathrm{h}}$. A small-angle X-ray powder diffractogram of this phase, recorded on dendron $\mathbf{I}$ (Fig. $1 \mathrm{~g}$ ), is shown in Fig. 2a. The synthesis of $\mathbf{I}$ is described in ref. 15 and Supplementary Information, where this compound is labelled $\left[3,4,5-(3,5)^{2}\right] 12 \mathrm{G}_{3} \mathrm{CH}_{2} \mathrm{OH}$. Other compounds that show the X-ray signature of this phase include $(4-3,4,5-3,5) 12 \mathrm{G}_{2} \mathrm{CH}_{2} \mathrm{OH},\left[4-(3,4,5)^{2}\right] 12 \mathrm{G}_{2} \mathrm{COOH},\left[3,4-(3,5)^{2}\right]$ $12 \mathrm{G}_{3} \mathrm{COOH},\left[3,4-(3,5)^{2}\right] 12 \mathrm{G}_{3} \mathrm{CH}_{2} \mathrm{OH},\left[3,4-(3,4,5)^{2}\right] 12 \mathrm{G}_{3} \mathrm{CH}_{2} \mathrm{OH}$ (ref. 15), polyoxazolines with tapered side groups containing alkyl chains of different lengths ${ }^{16}$, as well as certain salts of 3,4,5-tris-( $n$ alkoxy)benzoic acid ${ }^{17}$.

On heating, compound I shows the following phase sequence: room temperature $\rightarrow \mathrm{X} \rightarrow 71^{\circ} \mathrm{C} \rightarrow P 4_{2} / m n m \rightarrow 72^{\circ} \mathrm{C} \rightarrow$ isotropic liquid, while on cooling phase $\mathrm{X}$ forms directly from the liquid (Supplementary Information). This allowed us to grow monodomains of the unknown phase. That phase $\mathrm{X}$ is a quasicrystal is revealed by the distinctive but crystallographically forbidden 12 -fold symmetry of the small-angle X-ray single-crystal pattern (Fig. 2b). When the sample is rotated around the 12-fold axis with the incident beam perpendicular to the axis, the diffraction pattern repeats every $30^{\circ}$. One such pattern is shown in Fig. $2 \mathrm{c}$, where the Ewald sphere cuts through a pair of strong reflections in Fig. $2 \mathrm{~b}$. The structure of this liquid quasicrystal (LQC) is periodic in the direction of the 12-fold axis, but quasiperiodic in the plane perpendicular to it.

In contrast to normal 3D periodic structures, five instead of three basis vectors are needed for indexing the diffraction peaks of a dodecagonal quasicrystal ${ }^{18}$. Four of the vectors, $\mathbf{q}^{1}, \mathbf{q}^{2}, \mathbf{q}^{3}$ and $\mathbf{q}^{4}$,
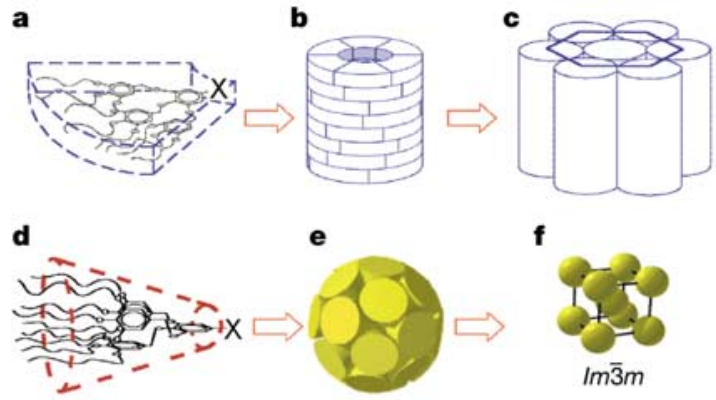

$\mathbf{f}$
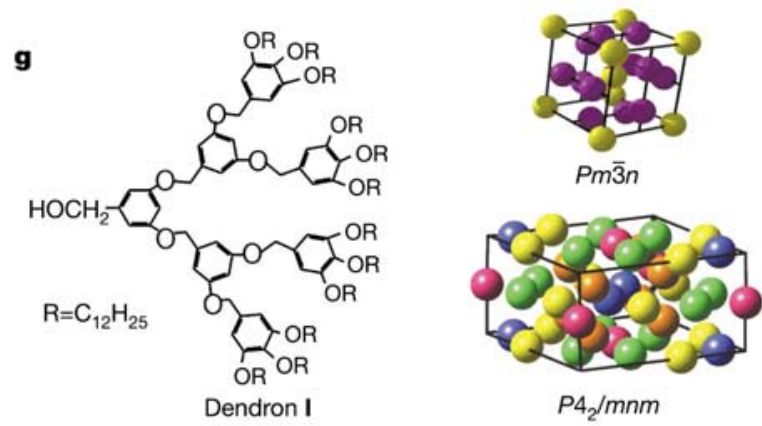

Figure 1 Self-assembly of wedge-shaped molecules. a, Dendrons with fewer tethered chains adopt a flat slice-like shape ( $X$ is a weakly binding group). $\mathbf{b}$, The slices stack up and form cylindrical columns, which assemble on a two-dimensional hexagonal columnar $\left(\mathrm{Co}_{\mathrm{h}}\right)$ lattice (c). d, Dendrons with more end-chains assume a conical shape. e, The cones assemble into spheres, which pack on three different 3D lattices $(\mathbf{f})$ with symmetries Im $\overline{3} m, P m \overline{3} n$ and $P 4_{2} / m n m$. g. Structure of compound I studied in this work. 